\title{
Analysis of the surgical benefits of 5-ALA-induced fluorescence in intracranial meningiomas: experience in 204 meningiomas
}

\author{
Matthias Millesi, MD, ${ }^{1,2}$ Barbara Kiesel, MD,,2 Mario Mischkulnig, ${ }^{1}$ \\ Mauricio Martínez-Moreno, MD, ${ }^{1}$ Adelheid Wöhrer, MD, PhD, ${ }^{2,3}$ Stefan Wolfsberger, MD, ${ }^{1,2}$ \\ Engelbert Knosp, MD,1,2 and Georg Widhalm, MD, PhD ${ }^{1,2}$ \\ ${ }^{1}$ Department of Neurosurgery, ${ }^{2}$ Comprehensive Cancer Center-Central Nervous System Tumors Unit, and ${ }^{3}$ Institute of
Neurology, Medical University of Vienna, Austria
}

OBJECTIVE One of the most important causes for recurrence of intracranial meningiomas is residual tumor tissue that remains despite assumed complete resection. Recently, intraoperative visualization of meningioma tissue by 5-aminolevulinic acid (5-ALA)-induced protoporphyrin IX (PpIX) fluorescence was reported. The aim of this study was to investigate the possible surgical benefits of PpIX fluorescence for detection of meningioma tissue.

METHODS 5-ALA was administered preoperatively to 190 patients undergoing resection of 204 intracranial meningiomas. The meningiomas' PpIX fluorescence status, fluorescence quality (strong or vague), and intratumoral fluorescence homogeneity were investigated during surgery. Additionally, specific sites, including the dural tail, tumor-infiltrated bone flap, adjacent cortex, and potential satellite lesions, were analyzed for PpIX fluorescence in selected cases.

RESULTS PpIX fluorescence was observed in 185 (91\%) of 204 meningiomas. In the subgroup of sphenoorbital meningiomas (12 of 204 cases), the dural part showed visible PpIX fluorescence in 9 cases (75\%), whereas the bony part did not show any PpIX fluorescence in 10 cases (83\%). Of all fluorescing meningiomas, 168 (91\%) showed strong PpIX fluorescence. Typically, most meningiomas demonstrated homogeneous fluorescence (75\% of cases). No PpIX fluorescence was observed in any of the investigated 89 dural tails. In contrast, satellite lesions could be identified through PpIX fluorescence in 7 cases. Furthermore, tumor-infiltrated bone flaps could be visualized by PpIX fluorescence in all 13 cases. Notably, PpIX fluorescence was also present in the adjacent cortex in 20 (25\%) of 80 analyzed cases.

CONCLUSIONS The authors' data from this largest patient cohort to date indicate that PpIX fluorescence enables intraoperatively visualization of most intracranial meningiomas and allows identification of residual tumor tissue at specific sites. Thus, intraoperative detection of residual meningioma tissue by PpIX fluorescence might in future reduce the risk of recurrence.

http://thejns.org/doi/abs/10.3171/2015.12.JNS151513

KEY WORDS meningioma; 5-ALA; PpIX fluorescence; bone invasion; satellite lesions; oncology

I NTRACRANIAL meningiomas are the most common primary central nervous system tumors, representing approximately $30 \%$ of these neoplasms. ${ }^{43}$ Complete resection of meningiomas is the treatment of choice in the vast majority of patients to minimize the risk of tumor recurrence. ${ }^{24}$ According to World Health Organization (WHO) criteria, WHO Grade II and III meningiomas show higher rates of recurrence than the frequently observed WHO Grade I meningiomas. ${ }^{9,43}$ In the long-term follow-up of surgically treated meningiomas, however, tumor recurrence is not uncommon, even in WHO Grade I meningiomas, and is reported in $24 \%-38 \%$ of cases. ${ }^{24,25,36}$

One of the most important reasons for recurrence of intracranial meningiomas is residual tumor tissue that remains unrecognized during resection. ${ }^{21,43}$ Several typical sites of residual meningioma tissue-and thus origins of tumor recurrence-have been noted. First, the typically observed "dural tail," consisting of a thickening of the 
dura mater adjacent to the meningioma that is a site of potential tumor infiltration, might lead to tumor recurrence. ${ }^{17,27,34}$ Second, small satellite lesions at a distance of up to $3 \mathrm{~cm}$ from the meningioma and tumor infiltration of the bone flap might be missed by the neurosurgeon. ${ }^{2,3,14}$ Finally, the adjacent cortex may harbor tumor cells, as shown by a recent report of the finding of tumor cells at the brain-tumor interface in high-grade meningiomas, ${ }^{7}$ representing another potential cause of incomplete resection. Therefore, techniques to visualize residual tumor tissue during meningioma surgery are warranted in order to minimize the rate of tumor recurrence.

In the last 2 decades, intraoperative visualization of malignant glioma tissue with assistance of the fluorescent dye 5-aminolevulinic acid (5-ALA) has been increasingly applied. ${ }^{33}$ Oral administration of 5-ALA prior to surgery leads to visible intratumoral protoporphyrin IX (PpIX) fluorescence during resection. ${ }^{32}$ In a multicenter Phase III trial, Stummer et al. ${ }^{30}$ demonstrated that rates of complete resections for malignant glioma were significantly higher with 5-ALA fluorescence-guided surgery than with conventional white-light procedures. Based on these encouraging data, 5-ALA was also applied in the management of other tumors and was considered useful, particularly for suspected low-grade gliomas, stereotactic brain tumor biopsies, and specific spinal neoplasms. 12,20,37,39-41 Recently, the presence of 5-ALA-induced PpIX fluorescence was also observed in intracranial meningiomas. ${ }^{1,4,5,7,8,10,11,13,16,18,21,22,35,38,42}$ However, the benefits of PpIX fluorescence in intracranial meningioma surgery had not been systematically analyzed in a large patient series.

The aim of the present study was therefore to systematically investigate the possible surgical benefits of the use of 5-ALA in a large cohort of patients with intracranial meningiomas. In our study, we analyzed these meningiomas for PpIX fluorescence status, fluorescence quality (strong or vague), and intratumoral fluorescence homogeneity. Furthermore, we examined the value of PpIX fluorescence for detection of residual tumor tissue at typical sites of meningioma recurrence.

\section{Methods}

This study included 190 consecutive patients who were treated for intracranial meningiomas between 2009 and 2013 at the Department of Neurosurgery of the Medical University of Vienna and received 5-ALA prior to resection. The study was approved by the local ethics committee and patients gave informed consent. Patient characteristics are shown in Table 1.

\section{Preoperative Imaging}

Each patient underwent diagnostic imaging with administration of contrast medium prior to hospitalization. MRI was performed in most cases (186 patients); CT was used for patients with a contraindication to MRI (4 patients). In cases of suspected bone flap infiltration on preoperative MRI, an additional CT was obtained. Within 1 week prior to surgery, a contrast-enhanced T1-weighted MRI study and/or contrast-enhanced CT was performed in order to acquire imaging data for the navigation system.
TABLE 1. Summary of clinical and demographic characteristics of the patient cohort

\begin{tabular}{cc}
\hline Characteristic & Value $^{*}$ \\
\hline No. of patients & 190 \\
\hline Sex ratio, F/M & $2.2: 1$ \\
\hline Age (yrs) & 57 \\
\hline Median & $24-88$ \\
\hline Range & $204(100)$ \\
\hline No. of meningiomas & $167(82)$ \\
\hline Newly diagnosed & $37(18)$ \\
\hline Recurrent & \\
\hline Simpson grade of resection & $91(45)$ \\
\hline I & $45(22)$ \\
\hline II & $35(17)$ \\
\hline III & $33(16)$ \\
\hline IV & $0(0)$ \\
\hline IV & $155(76)$ \\
\hline Histological diagnosis & $104(67)$ \\
\hline WHO Grade I & $28(18)$ \\
\hline Meningothelial & $12(8)$ \\
\hline Transitional & $4(3)$ \\
\hline Fibrous & $2(1)$ \\
\hline Secretory & $2(1)$ \\
\hline Psammomatous & $2(1)$ \\
\hline Microcystic & $1(1)$ \\
\hline Angiomatous & $33(16)$ \\
\hline NOS & $29(88)$ \\
\hline AHO Grade II & $3(9)$ \\
\hline Chorent numbers of tumors (\%) otherwise indicated.
\end{tabular}

\section{Neurosurgical Meningioma Resection}

The resection of all meningiomas was performed under conventional white-light microscopy with the assistance of a navigation system. The extent of resection of each meningioma was classified intraoperatively by the operating neurosurgeon according to the Simpson classification (see Table 2). ${ }^{28}$ Additionally, the existence of an arachnoid plane for surgical dissection (intact or disrupted) was analyzed in all cases.

\section{Analysis of 5-ALA-Induced PpIX Fluorescence}

Oral solutions of 5-ALA (20 mg/kg body weight) were administered in all patients approximately 3 hours before induction of anesthesia. During resection, each meningioma was checked repeatedly for potential PpIX fluorescence using a modified neurosurgical microscope (NC4 or Pentero, Carl Zeiss Surgical GmbH) with the ability to switch to violet-blue excitation light. ${ }^{31}$ 
TABLE 2. Simpson classification

\begin{tabular}{cc}
\hline EOR & \multicolumn{1}{c}{ Surgical Outcome } \\
\hline Grade I & $\begin{array}{c}\text { Macroscopic complete removal of tumor with excision of } \\
\text { dural attachment and abnormal bone }\end{array}$ \\
\hline Grade II & $\begin{array}{c}\text { Macroscopic complete removal of tumor with coagulation } \\
\text { of dural attachment }\end{array}$ \\
\hline Grade III & $\begin{array}{c}\text { Macroscopic complete removal of tumor without removal/ } \\
\text { coagulation of dural attachment }\end{array}$ \\
\hline Grade IV & Partial resection \\
\hline Grade V & Decompression with or without biopsy \\
\hline
\end{tabular}

$\mathrm{EOR}=$ extent of resection.

The following specific aspects were analyzed during resection.

\section{PpIX Fluorescence Status, Fluorescence Quality, and}

Intratumoral Fluorescence Homogeneity

In each meningioma, the intraoperative PpIX fluorescence status was checked and documented as positive or negative. In fluorescing meningiomas, the PpIX fluorescence quality was classified semiquantitatively as strong or vague and the intratumoral fluorescence homogeneity was categorized as homogeneous or inhomogeneous, as described previously. ${ }^{20,39-41}$ In sphenoorbital meningiomas, the infiltrated bone as well as the dural part were investigated separately for P PIX fluorescence.

\section{PpIX Fluorescence at Specific Sites of Potential Tumor Recurrence}

We investigated typical sites of potential meningioma recurrence for PpIX fluorescence during surgery. These sites included the dural tail as detected by MRI, potential satellite lesions in proximity of the resected meningioma, and radiologically confirmed tumor infiltration of the bone flap. Because we also observed PpIX fluorescence in the adjacent cortex of 1 meningioma in the course of the study, we included this site in our analysis in subsequent cases-analyzing the surrounding cortex for PpIX fluorescence during and after the meningioma resection.

\section{Postoperative Course and Follow-Up}

All patients were protected from natural light sources for 24 hours after 5-ALA administration due to the possible phototoxic effect of this fluorescent dye. To detect new postoperative deficits, a neurological assessment was performed in each patient prior to surgery as well as before discharge and during the first follow-up visit approximately 3 months after surgery. A new deficit was considered permanent if it was still present at the first follow-up visit. In contrast, a neurological deficit was considered transient if the symptoms had resolved by the first follow-up visit.

\section{Histopathology}

All tumors and other tissue samples were routinely processed for histopathological analysis, and histopathological diagnosis was established according to the current WHO criteria. ${ }^{9}$

\section{Statistical Analysis}

For statistical analysis, we used SPSS 21.0 statistical software (IBM Corp.). A Jonckheere-Terpstra test was calculated for the variables PpIX fluorescence and WHO grade. For qualitative variables, a chi-square test was performed. A p value $<0.05$ was considered statistically significant.

\section{Results}

The intraoperative application of the 5-ALA-based technology was feasible in all 190 patients with a total of 204 intracranial meningiomas, and no relevant side effects related to 5-ALA occurred.

\section{Patient Characteristics}

The median age of our patient cohort was 57 years (range 24-88 years). The group included 131 women and 59 men. The most common meningioma localizations were the convexity (52 [25\%] of 204 tumors), the parasagittal region (32 tumors [16\%]), and the sphenoid wing (20 tumors [10\%]). Histopathological examination revealed a WHO Grade I meningioma in 155 cases (76\%), a WHO Grade II meningioma in 33 cases $(16 \%)$, and a WHO Grade III meningioma in 16 cases (8\%). Further details are given in Tables 1 and 3.

\section{Neurosurgical Meningioma Resection}

A total of 180 patients each underwent a single procedure; in 178 of these patients, a single meningioma was resected, and in the other 2 patients, 2 meningiomas were resected during a single operation. Five patients with multiple meningiomas underwent 2 separate operations for removal of 2 meningiomas at distant locations. Four patients with high-grade meningiomas (2 WHO Grade II and 2 WHO Grade III) developed tumor recurrence and underwent an additional resection, so each of these patients also underwent 2 operations. Finally, 1 patient with a WHO Grade III meningioma underwent 3 additional operations due to local tumor recurrence and CSF spreading after primary resection (i.e., a total of 4 operations overall).

\section{Extent of Resection}

The extent of resections achieved according to Simpson Grade were as follows: Simpson Grade I in 91 tumors (45\%), Simpson Grade II in 45 tumors (22\%), Simpson Grade III in 35 tumors (17\%), and Simpson Grade IV in 33 tumors $(16 \%)$.

\section{Meningioma Arachnoid Status}

In each meningioma with an extraventricular location (202 tumors), the existence of an arachnoidal dissection plane was analyzed intraoperatively. We found that the arachnoid layer was disrupted in 89 of these cases (44\%). In contrast, an intact dissection plane was present in the remaining 113 cases (56\%).

\section{Intraoperative 5-ALA-Induced PpIX Fluorescence}

Each meningioma was checked repeatedly for 5-ALAinduced PpIX fluorescence during resection (see also Table 3 and Fig. 1). 
TABLE 3. Overview of the PpIX fluorescence status, fluorescence quality, and intratumoral fluorescence homogeneity according to the intracranial meningioma localization and WHO grade*

\begin{tabular}{|c|c|c|c|c|c|c|c|}
\hline \multirow[b]{2}{*}{ Characteristic } & \multirow[b]{2}{*}{ All Tumors } & \multicolumn{2}{|c|}{ Fluorescence Status } & \multicolumn{2}{|c|}{ Fluorescence Quality } & \multicolumn{2}{|c|}{ Fluorescence Homogeneity } \\
\hline & & Pos & $\mathrm{Neg}$ & Strong & Vague & Homogen & Inhomogen \\
\hline \multicolumn{8}{|l|}{ Location } \\
\hline Convexity & $52(25)$ & $48(92)$ & $4(8)$ & $41(85)$ & $7(15)$ & $32(80)$ & $8(20)$ \\
\hline Falx/parasagittal & $44(22)$ & $43(98)$ & $1(2)$ & $42(98)$ & $1(2)$ & $29(73)$ & $11(27)$ \\
\hline Anterior cranial fossa & $63(31)$ & $56(89)$ & $7(11)$ & $51(91)$ & $5(9)$ & $32(78)$ & $9(22)$ \\
\hline Sphenoid wing & $30(15)$ & $27(90)$ & $3(10)$ & $25(93)$ & $2(7)$ & $16(92)$ & $2(8)$ \\
\hline Sphenoorbital & $12(6)$ & $9(75)$ & $3(25)$ & $8(89)$ & $1(11)$ & $6(75)$ & $2(25)$ \\
\hline Infiltrated bone & & $2(17)$ & $10(83)$ & $2(100)$ & $0(0)$ & $2(100)$ & $0(0)$ \\
\hline Dural part & & $9(75)$ & $3(25)$ & $8(89)$ & $1(11)$ & $6(75)$ & $2(25)$ \\
\hline Tuberculum sellae & $10(5)$ & $9(90)$ & $1(10)$ & $8(89)$ & $1(11)$ & $5(83)$ & $1(17)$ \\
\hline Olfactory groove & $5(2)$ & $5(100)$ & $0(0)$ & $4(80)$ & $1(20)$ & $1(33)$ & $2(67)$ \\
\hline Frontobasal & $3(1)$ & $3(100)$ & $0(0)$ & $3(100)$ & $0(0)$ & $2(100)$ & $0(0)$ \\
\hline Planum sphenoidale & $3(1)$ & $3(100)$ & $0(0)$ & $3(100)$ & $0(0)$ & $2(100)$ & $0(0)$ \\
\hline Middle cranial fossa & $10(5)$ & $9(90)$ & $1(10)$ & $7(78)$ & $2(22)$ & $2(33)$ & $4(67)$ \\
\hline Middle fossa floor & $6(3)$ & $5(83)$ & $1(17)$ & $5(100)$ & $0(0)$ & $2(50)$ & $2(50)$ \\
\hline Cavernous sinus & $4(2)$ & $4(100)$ & $0(0)$ & $2(50)$ & $2(50)$ & $0(0)$ & $2(100)$ \\
\hline Posterior cranial fossa & $33(16)$ & $27(82)$ & $6(18)$ & $25(93)$ & $2(7)$ & $16(70)$ & $7(30)$ \\
\hline Petroclival & $8(4)$ & $8(100)$ & $0(0)$ & $8(100)$ & $0(0)$ & $5(62)$ & $3(38)$ \\
\hline Petrous bone & $8(4)$ & $7(88)$ & $1(12)$ & $7(100)$ & $0(0)$ & $4(80)$ & $1(20)$ \\
\hline Foramen magnum & $6(3)$ & $5(83)$ & $1(17)$ & $4(80)$ & $1(20)$ & $3(60)$ & $2(40)$ \\
\hline Tentorium & $7(4)$ & $5(71)$ & $2(29)$ & $4(80)$ & $1(20)$ & $3(100)$ & $0(0)$ \\
\hline Posterior fossa convexity & $2(1)$ & $1(50)$ & $1(50)$ & $1(100)$ & $0(0)$ & $0(0)$ & $1(100)$ \\
\hline Clivus & $2(1)$ & $1(50)$ & $1(50)$ & $1(100)$ & $0(0)$ & $1(100)$ & $0(0)$ \\
\hline Intraventricular & $2(1)$ & $2(100)$ & $0(0)$ & $2(100)$ & $0(0)$ & $2(100)$ & $0(0)$ \\
\hline \multicolumn{8}{|l|}{ WHO grade } \\
\hline I & $155(76)$ & $139(90)$ & $16(10)$ & $124(89)$ & $15(11)$ & $81(74)$ & $28(26)$ \\
\hline II & $33(16)$ & 30 (91) & $3(9)$ & $28(93)$ & $2(7)$ & $19(73)$ & $7(27)$ \\
\hline III & $16(8)$ & $16(100)$ & $0(0)$ & $16(100)$ & $0(0)$ & $13(87)$ & $2(13)$ \\
\hline
\end{tabular}

Homogen = homogeneous; inhomogen = inhomogeneous; neg = negative; pos = positive.

* Values represent number of tumors (\%).

\section{PpIX Fluorescence Status and Fluorescence Quality}

PpIX fluorescence was detected in 185 (91\%) of the 204 resected meningiomas. Of these, 168 cases (91\%) showed strong PpIX fluorescence and 17 cases $(9 \%)$ showed vague PpIX fluorescence. No fluorescence was detected in any part of 19 meningiomas (9\%).

\section{Intratumoral Fluorescence Homogeneity}

The homogeneity of PpIX fluorescence was evaluated in 150 of the 185 fluorescing meningiomas. In 113 (75\%) of these tumors, a homogeneous PpIX fluorescence was observed, whereas 37 tumors (25\%) showed an inhomogeneous fluorescence pattern.

\section{PpIX Fluorescence and Tumor Localization}

We observed PpIX fluorescence (strong or vague) in 43 (98\%) of 44 falx/parasagittal meningiomas, 48 (92\%) of 52 convexity meningiomas, $9(90 \%)$ of 10 middle cranial fossa meningiomas, 56 (89\%) of 63 anterior cranial fossa meningiomas, and $27(82 \%)$ of 33 posterior cranial fossa meningiomas. Furthermore, both intraventricular meningiomas showed PpIX fluorescence. We did not find a significant correlation between the presence of PpIX fluorescence and the localization of the intracranial meningioma.

Because 2 different compartments (infiltrated bone and the dural part) exist in sphenoorbital meningiomas, these compartments were investigated separately in these lesions $(\mathrm{n}=12)$. Within the infiltrated bone, no PpIX fluorescence was detected in most cases (10 cases [83\%]). In contrast, the dural part showed PpIX fluorescence in most cases ( 9 cases [75\%]).

\section{PpIX Fluorescence and WHO Grade}

We observed PpIX fluorescence (strong or vague) in 139 (90\%) of 155 WHO Grade I meningiomas, in 30 (91\%) of 33 WHO Grade II meningiomas, and in all 16 WHO Grade III meningiomas (100\%). We did not find a statistically significant relationship between PpIX fluorescence status $(\mathrm{p}=0.092)$ or fluorescence quality (strong or vague, $\mathrm{p}=0.191)$ and $\mathrm{WHO}$ grade . 

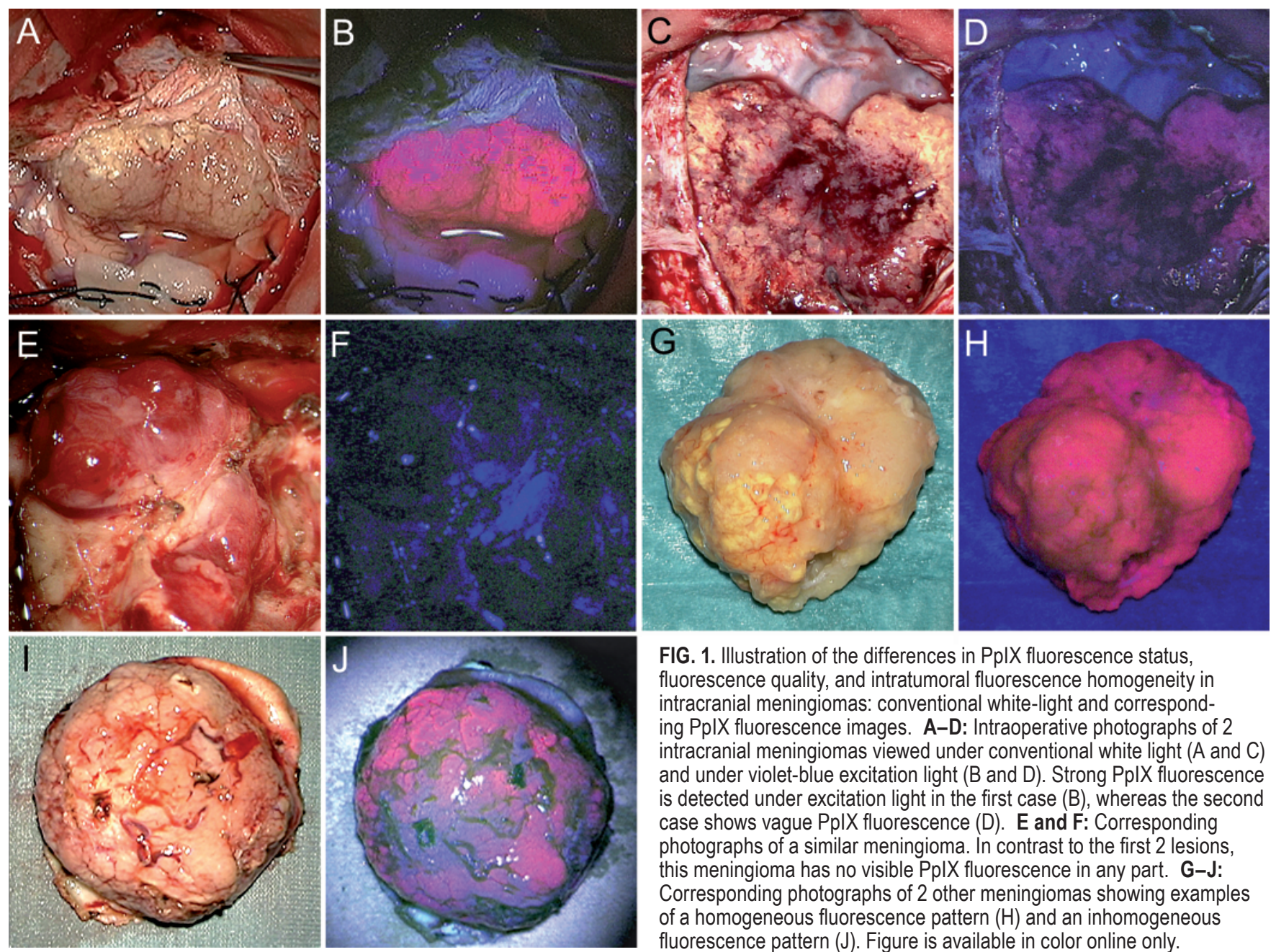

FIG. 1. Illustration of the differences in PpIX fluorescence status, fluorescence quality, and intratumoral fluorescence homogeneity in intracranial meningiomas: conventional white-light and corresponding PpIX fluorescence images. A-D: Intraoperative photographs of 2 intracranial meningiomas viewed under conventional white light ( $A$ and $C$ ) and under violet-blue excitation light (B and D). Strong PpIX fluorescence is detected under excitation light in the first case $(B)$, whereas the second case shows vague PpIX fluorescence (D). E and F: Corresponding photographs of a similar meningioma. In contrast to the first 2 lesions, this meningioma has no visible PpIX fluorescence in any part. G-J: Corresponding photographs of 2 other meningiomas showing examples of a homogeneous fluorescence pattern $(\mathrm{H})$ and an inhomogeneous fluorescence pattern $(\mathrm{J})$. Figure is available in color online only.

\section{PpIX Fluorescence at Specific Sites of Potential Meningioma Recurrence}

During surgery, typical sites that might result in meningioma recurrence were investigated for PpIX fluorescence (see also Fig. 2).

\section{Dural Tail}

In the first 89 cases with a dural tail detectable on MRI, we systematically analyzed this specific site for PpIX fluorescence. No PpIX fluorescence was detected in any of these cases. Consequently, we abandoned investigation of this feature in subsequent cases. Separate samples of nonfluorescing dural tails were available for histopathological analysis in 16 cases; no obvious tumor cells were found in $11(69 \%)$ of these cases and distinct tumor cells were found in 5 cases $(31 \%)$.

\section{Satellite Lesions}

In all cases, we used PpIX fluorescence to assess for the presence of potential satellite lesions distant to the meningioma. Altogether, we identified 7 small fluorescing meningioma satellites that were barely recognizable with conventional white light alone. The small fluoresc-

ing satellite lesions were not identified on the preoperative imaging in any of these cases.

\section{Infiltrated Bone Flap}

In cases in which preoperative imaging showed bone flap infiltration, the bone flap was investigated for PpIX fluorescence (12 convexity/parasagittal meningiomas and 1 sphenoid wing meningioma). In all of these cases, the tumor infiltration into the bone was visualized by PpIX fluorescence. In all cases in which separate tissue samples were available for analysis (7 cases), tumor infiltration of the fluorescing bone flap was confirmed by histopathological analyses.

\section{Adjacent Cortex}

During this study, we were surprised by the observation of PpIX fluorescence in the cortex adjacent to a WHO Grade III meningioma. Based on this observation, we included the adjacent cortex for investigation of PpIX fluorescence in subsequent cases (altogether 80 cases including the first patient) because this site might be an additional origin of meningioma recurrence. PpIX fluorescence was detected in the adjacent cortex in $20(25 \%)$ of these 80 cases; 60 cases (75\%) did not show any PpIX fluorescence. 

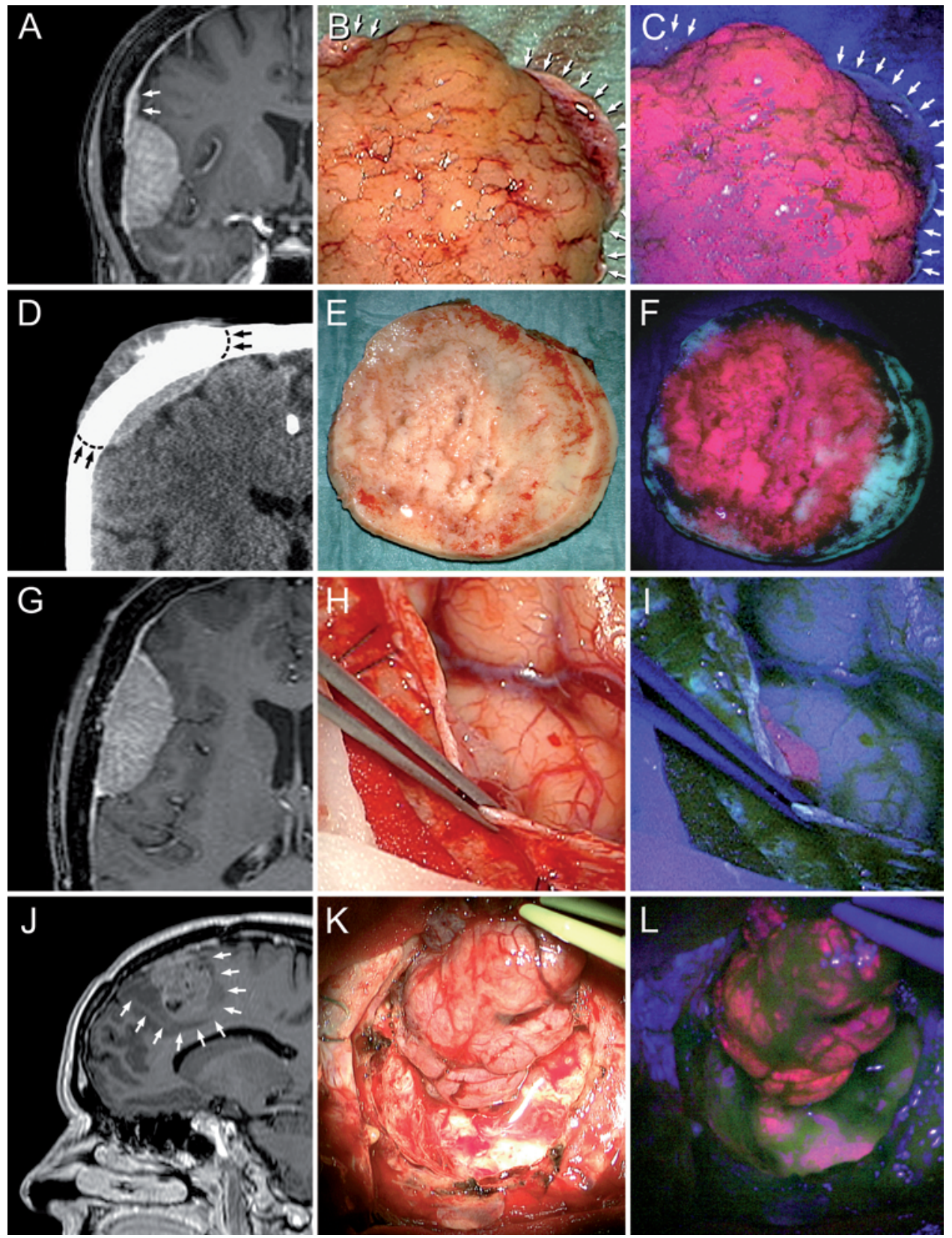

FIG. 2. Illustration of different PpIX fluorescence effects at specific intraoperative sites of potential meningioma recurrence: preoperative imaging, conventional white-light, and corresponding PpIX fluorescence. A-C: Dural tail. Preoperative contrast-enhanced T1-weighted MRI (coronal image in A) and conventional white-light microscopy (B) demonstrate the dural tail (white arrows), which cannot be visualized by PpIX fluorescence $(C)$, although the meningioma shows strong PpIX fluorescence. D-F: Bone flap. In another meningioma with radiological evidence of bone flap infiltration (black arrows in D, coronal CT image), the suspected bone invasion (shown under conventional white light in E) can be clearly visualized by visible PpIX fluorescence (F). G-I: Satellite lesion. A small satellite lesion distant to the meningioma is not visible on MRI (G, contrast-enhanced T1-weighted axial image) and is barely recognizable under white-light microscopy $(\mathrm{H})$, but can be clearly identified by visible PpIX fluorescence (I). J-L: Adjacent cortex. In the region of the peritumoral edema (white arrows in J, contrast-enhanced T1-weighted sagittal MR image) of a WHO Grade III meningioma, adjacent cortex that appeared normal under conventional white-light microscopy (K) demonstrates visible $\mathrm{PpIX}$ fluorescence (L). Figure is available in color online only.

\section{PpIX Fluorescence of Adjacent Cortex and WHO} Grade. Moreover, we analyzed the occurrence of PpIX fluorescence in the adjacent cortex of these 80 cases with respect to WHO grade. Visible PpIX fluorescence in the adjacent cortex was observed more often in WHO Grade III meningiomas (55\%) than in WHO Grade I or II menin- giomas (21\% and $18 \%$, respectively). However, this difference did not reach statistical significance $(p=0.132)$. For further details see Table 4.

PpIX Fluorescence of Adjacent Cortex and Arachnoid Status. Additionally, we correlated the PpIX fluorescence of the adjacent cortex with the meningioma arachnoid sta- 
tus. We found a significantly higher proportion of PpIX fluorescence within the adjacent cortex in cases with a disrupted arachnoid layer than in cases with an intact arachnoid layer (41\% vs $11 \%$; $p=0.002)$. Finally, we performed a subgroup analysis of the fluorescence status in the adjacent cortex in cases with and without disruption of the arachnoid layer and according to the WHO grade. This analysis did not reveal any statistically significant differences $(p=0.525$ and $p=0.101$, respectively). Further details are provided in Table 4.

\section{Postoperative Outcome}

In the total of 202 procedures, there was 1 case of perioperative mortality due to pulmonary embolism 3 days after resection of a large medial sphenoid wing meningioma. One other patient, who underwent resection of a large clival meningioma and had worsening of preexisting tetraparesis and a severe swallowing deficit, died 3 months after surgery due to pneumonia. Additionally, new neurological deficits occurred in 13 patients. In 5 cases, the deficits were transient and had resolved by the first follow-up visit approximately 3 months after surgery. In the other 8 cases, the deficits were permanent.

\section{Discussion}

In the current study, we systematically investigated the possible surgical benefits of 5-ALA-induced PpIX fluorescence in the largest series of intracranial meningiomas reported to date. According to our data on 204 meningiomas, PpIX fluorescence provided intraoperative visualization of such tumors in the majority of cases. Furthermore, PpIX fluorescence was also useful for visualizing tumor tissue at specific sites of potential meningioma recurrence.

\section{Recurrence of Intracranial Meningiomas}

In 1957, Donald Simpson had already identified the extent of resection as one of the key factors for tumor recurrence and prognosis in patients suffering from intracranial meningiomas. ${ }^{28}$ In this study by Simpson, a considerably lower meningioma recurrence was observed in cases in which complete tumor resection was achieved with excision of the adjacent dura and abnormal bone (Simpson Grade I) than in cases in which the adjacent dura was only coagulated (Simpson Grade II). ${ }^{28}$ Consequently, complete tumor resection is nowadays considered the treatment of choice for the vast majority of meningiomas. ${ }^{24}$ In the longterm follow-up, however, the rate of recurrence of intracranial meningiomas is reported as being as high as $38 \%$ despite complete resection under conventional white-light microscopy. ${ }^{25}$ One of the most important factors for meningioma recurrence is residual tumor tissue at specific intraoperative sites, such as the dural tail, adjacent bone flap, or brain, and potential satellite lesions. ${ }^{2,3,7,14,17,21,27,34}$ To minimize recurrence of intracranial meningiomas, intraoperative tools to identify residual tumor tissue are required.

\section{PpIX Fluorescence in Intracranial Meningiomas}

Intraoperative visualization of malignant glioma tissue
TABLE 4. Correlation of the PpIX fluorescence status in the adjacent cortex with WHO grade and meningioma arachnoid status*

\begin{tabular}{|c|c|c|c|c|}
\hline \multirow[b]{2}{*}{ Characteristic } & \multirow{2}{*}{$\begin{array}{l}\text { No. of } \\
\text { Tumors }\end{array}$} & \multicolumn{2}{|c|}{ Fluorescing Cortex } & \multirow[b]{2}{*}{ p Value } \\
\hline & & Yes & No & \\
\hline WHO grade & & & & 0.132 \\
\hline 1 & 52 & $11(21)$ & $41(79)$ & \\
\hline$\|$ & 17 & $3(18)$ & $14(82)$ & \\
\hline II & 11 & $6(55)$ & $5(45)$ & \\
\hline Arachnoid layer & & & & 0.002 \\
\hline Intact & 44 & $5(11)$ & $39(89)$ & \\
\hline Disrupted & 34 & $14(41)$ & $20(59)$ & \\
\hline Arachnoid layer intact & & & & 0.525 \\
\hline WHO Grade I & 30 & $4(13)$ & $26(87)$ & \\
\hline WHO Grade II & 12 & $1(8)$ & $11(92)$ & \\
\hline WHO Grade III & 2 & $0(0)$ & $2(100)$ & \\
\hline Arachnoid layer disrupted & & & & 0.101 \\
\hline WHO Grade I & 22 & $7(32)$ & $15(68)$ & \\
\hline WHO Grade II & 3 & $1(33)$ & $2(67)$ & \\
\hline WHO Grade III & 9 & $6(67)$ & $3(33)$ & \\
\hline
\end{tabular}

by fluorescence has become a promising approach to optimize resections in neurosurgery. ${ }^{29,33}$ In recent years, 5-ALA has also been administered to patients with intracranial meningiomas (see Table 5). In 2007, Kajimoto et al. ${ }^{16}$ first described the presence of visible PpIX fluorescence in intracranial meningioma tissue during resection. In 2008, Morofuji et al. ${ }^{21}$ observed PpIX fluorescence in the adjacent bone flap while resecting an intracranial meningioma. Further cases of intracranial meningiomas showing PpIX fluorescence were subsequently described. ${ }^{1,4,8,11,13,22,38,42}$ Recently, the first patient series investigating the use of 5-ALA in intracranial meningioma resection were reported. $5,7,10,18,35$ However, a systematic analysis of the surgical benefits of PpIX fluorescence in intracranial meningiomas in a large patient cohort has been missing.

\section{Present Study}

In this study comprising 204 intracranial meningiomas, we systematically analyzed the PpIX fluorescence status, fluorescence quality, and intratumoral fluorescence homogeneity as well as the value of PpIX fluorescence for detection of residual tumor tissue that might have remained at specific intraoperative sites. Images from a representative case, showing the use of 5-ALA in the resection of a tuberculum sellae meningioma, are provided in Fig. 3.

\section{PpIX Fluorescence Status and Fluorescence Quality}

We observed PpIX fluorescence in the vast majority of meningiomas (91\% of cases) during resection. In contrast, no visible PpIX fluorescence could be detected in the remaining $9 \%$ of cases. This is in accordance with the other 3 largest patient series, which reported PpIX fluorescence in $77 \%-94 \%$ of cases . $^{5,718}$ 


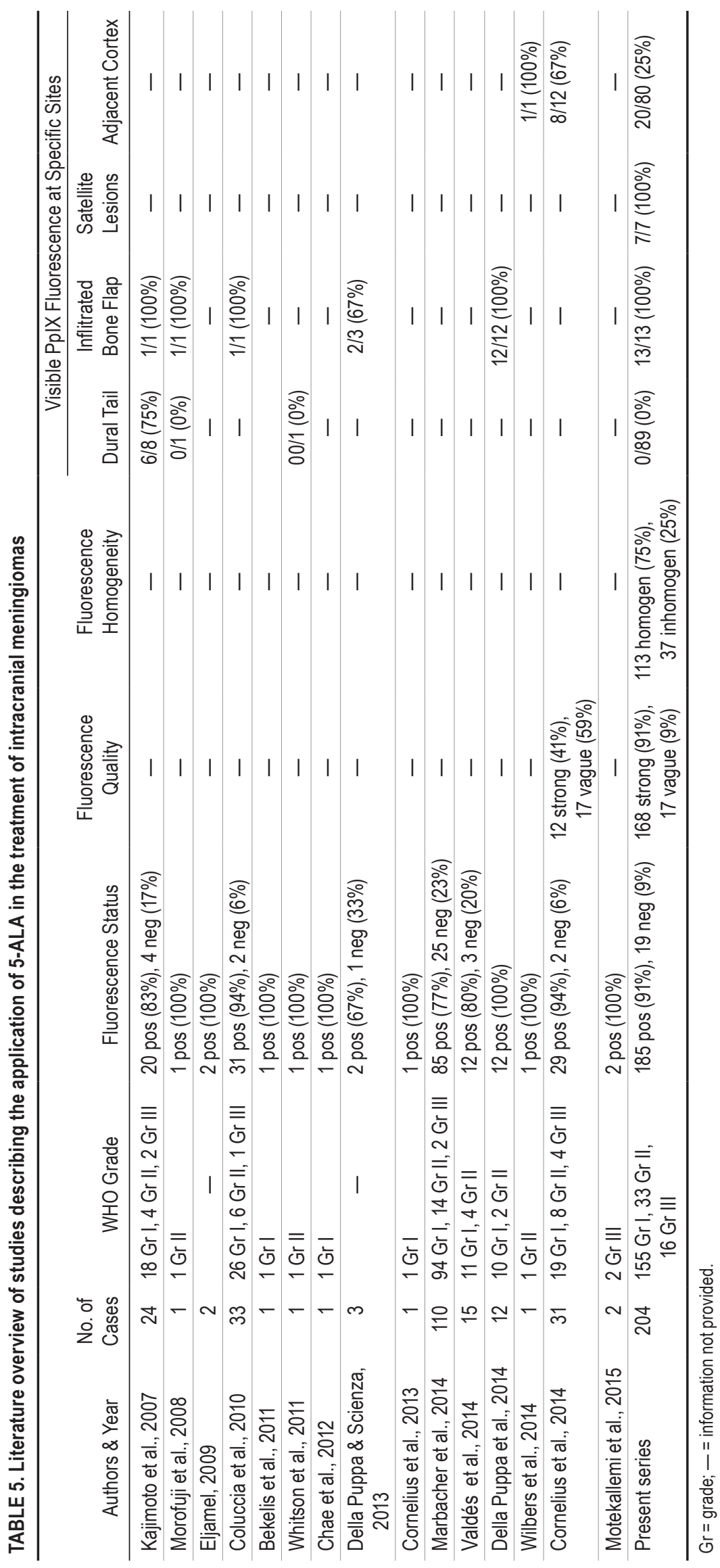


One possible explanation for the presence or absence of PpIX fluorescence in certain meningiomas might be related to differences in the metabolism of 5-ALA within the heme biosynthesis pathway. In this context, Hefti et al. ${ }^{15}$ observed that 5-ALA-induced PpIX accumulation in meningioma cells depends on the activity of ferrochelatase, which converts fluorescing PpIX to nonfluorescing heme. In their study, the authors described a meningioma cell line (HBL-52) with high activity of ferrochelatase showing low levels of PpIX accumulation compared with another cell line (BEN-MEN-1) with low activity of ferrochelatase demonstrating high levels of PpIX accumulation. Another possible explanation for the presence or absence of PpIX fluorescence might be differences on the molecular level between specific meningiomas, such as loss of heterozygosity of the NF2 gene on chromosome 22 or the activation of telomerase. ${ }^{6}$

Furthermore, we also classified the PpIX fluorescence quality as either strong or vague and typically found strong PpIX fluorescence in most meningiomas ( $91 \%$ of cases). So far, this feature was systematically analyzed only in 1 small case series by Cornelius et al., ${ }^{7}$ who observed a higher proportion of meningiomas with vague PpIX fluorescence (59\% of cases) than we found. In our study and also in the literature, no correlation between the presence of PpIX fluorescence or fluorescence quality and WHO grade was detected. ${ }^{5,18}$ The only exception was the study by Cornelius et al., ${ }^{7}$ who found a significantly higher percentage of strong PpIX fluorescence in high-grade meningiomas (WHO Grade II and III).

\section{Intratumoral Fluorescence Homogeneity}

In contrast to the frequently observed inhomogeneous strong as well as vague PpIX fluorescence of malignant gliomas, we typically found a homogeneous PpIX fluorescence pattern in most meningiomas (75\% of cases). To the best of our knowledge, this is the first study to systematically analyze this feature in a large patient cohort.

\section{Dural Tail}

No PpIX fluorescence was observed in the dural tail of any of the 89 meningiomas in which it was investigated. This is in accordance with the observations described by Morofuji et al. ${ }^{21}$ and Whitson et al. ${ }^{38}$ In contrast, Kajimoto et al. ${ }^{16}$ reported PpIX fluorescence in the dural tail in 6 of 8 cases. Interestingly, the authors found infiltration by tumor cells in 5 of these 6 fluorescing dural tails. ${ }^{16}$ Although none of the dural tails showed PpIX fluorescence in our study, tumor cells were detected by histopathological analyses in 5 of 16 analyzed cases. The exact morphological composition of the dural tail is still not fully clarified (tumor infiltration vs venous congestion). ${ }^{17,27}$ Although meningioma cells were found in some histopathologically analyzed dural tails in our study, the number and density of the tumor cells might have been too low to induce visible PpIX fluorescence.

\section{Satellite Lesions}

PpIX fluorescence was also useful in identifying 7 small satellite lesions distant from the meningioma that were barely recognizable with conventional white light
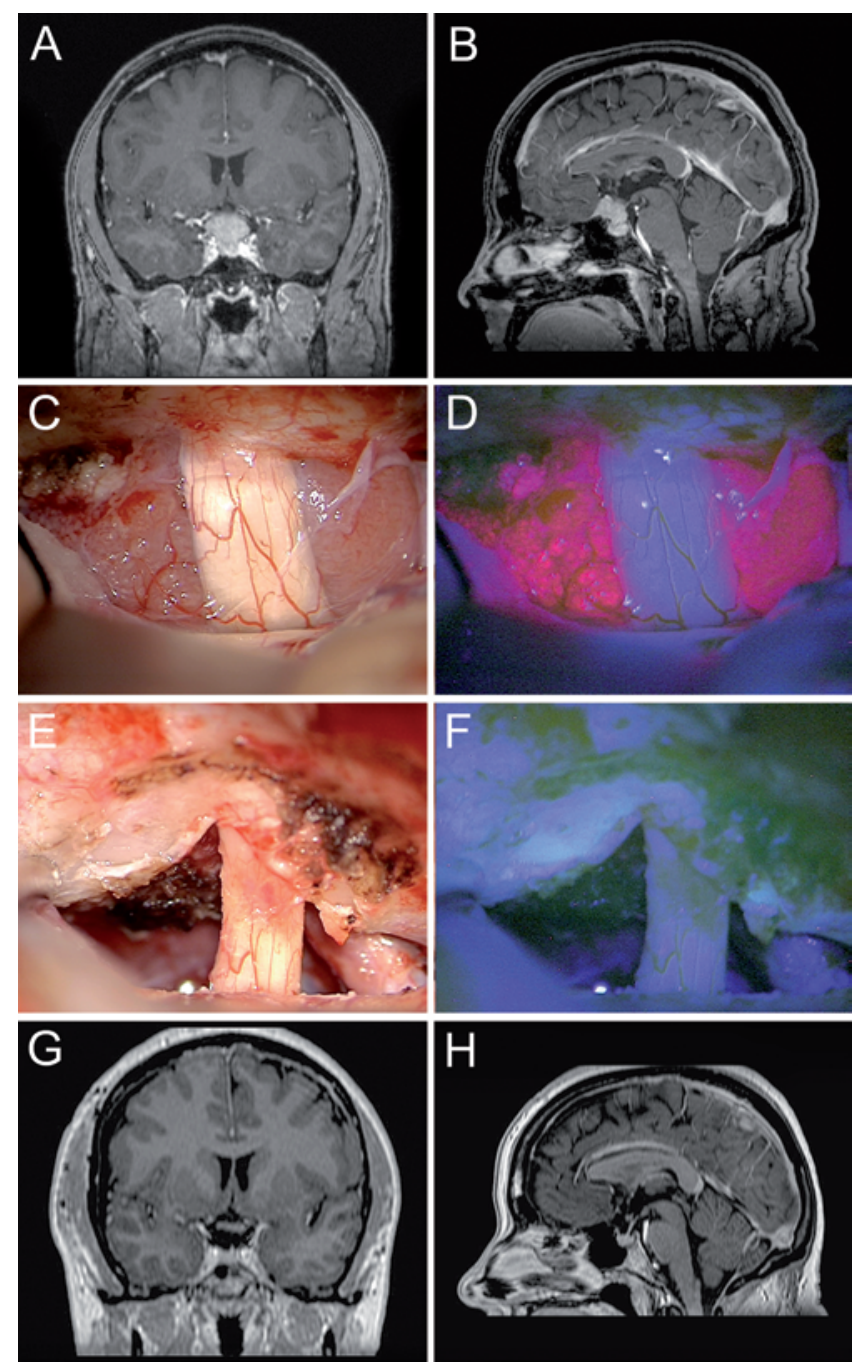

FIG. 3. Representative case of a patient with a tuberculum sellae meningioma presenting with visual disturbances who received 5-ALA prior to resection. A and B: Preoperative coronal $(A)$ and sagittal $(B)$ T1weighted MR images demonstrating a contrast-enhancing lesion at the tuberculum sellae with optic nerve compression. C: Intraoperative image obtained after a subfrontal approach, with the meningioma appearing behind the compressed optic nerve under white-light microscopy. D: Image obtained after changing to violet-blue excitation light, with the lesion clearly visualized by strong and homogeneous PpIX fluorescence (histology: WHO Grade I meningioma). E and F: Images obtained after complete tumor resection and opening of the optic canal. No remaining fluorescing tumor tissue can be detected at the end of surgery. $\mathbf{G}$ and H: Postoperative coronal $(\mathrm{G})$ and sagittal $(\mathrm{H})$ contrast-enhanced T1weighted MR images confirming complete resection of the meningioma with optic nerve decompression, which resulted in complete resolution of the visual disturbances. Figure is available in color online only.

alone and not visible on preoperative imaging. To the best of our knowledge, this is the first report of fluorescing satellite lesions in intracranial meningiomas.

\section{Infiltrated Bone Flap}

We found PpIX fluorescence in all 13 cases with bone flap infiltration, and tumor cells were detected by histopathology in all analyzed cases. Similar observations were described in the literature in single cases..$^{5,11,16,21}$ Recently, 
Della Puppa et al. ${ }^{10}$ investigated the value of PpIX fluorescence in areas of bone or bone flap invasion in 12 meningiomas. In each of these cases, PpIX fluorescence was observed, and histopathological examination detected tumor tissue in all fluorescing samples..$^{10}$

In contrast to the usually fluorescing bone flaps in our study, the bony part of our sphenoorbital meningiomas showed visible PpIX fluorescence in only 2 of 12 cases (17\%). Visualization of the bone involvement in meningiomas is of major importance in skull base surgery, as Pieper et al. reported tumor infiltration in hyperostotic bone in 35 of 51 cases. ${ }^{26}$ According to our data, however, the 5-ALA fluorescence technique is unfortunately not useful for visualizing the bony part in most sphenoorbital meningiomas. One possible explanation for the presence of PpIX fluorescence in bone flaps and the absence in the bony part of sphenoorbital meningiomas might be differences in growth patterns of specific meningiomas. The infiltration of the bone flap potentially originates from soft tumor invasion of the main meningioma bulk into the adjacent bone. In contrast, the bony part in sphenoorbital meningiomas usually represents a primary intraosseous/hyperostotic growth pattern. Furthermore, we cannot exclude that the absence of visible PpIX fluorescence in the bony part of sphenoorbital meningiomas is mainly caused by thermal injury caused by the high-speed drill system during tumor resection.

\section{Adjacent Cortex}

Interestingly, we observed PpIX fluorescence in the adjacent cortex in $25 \%$ of analyzed cases. Although fluorescing adjacent cortex was found more frequently in WHO Grade III meningiomas (55\%) than in WHO Grade I $(21 \%)$ or WHO Grade II meningiomas (18\%), this difference did not reach statistical significance. One possible explanation for visible PpIX fluorescence within the adjacent cortex might be leakage of 5-ALA/PpIX from the meningioma into the extracellular space, resulting in PpIX fluorescence in the surrounding brain tissue. ${ }^{19,23} \mathrm{On}$ the other hand, PpIX fluorescence of the adjacent cortex might also be caused by infiltration of the brain-tumor interface with meningioma cells. In this sense, Cornelius et al. ${ }^{7}$ observed PpIX fluorescence in the adjacent cortex in 8 of 12 high-grade meningiomas, and biopsy specimens obtained from this fluorescing adjacent cortex revealed tumor cells in all cases. Additionally, Wilbers et al. ${ }^{42}$ described 1 case of a recurrent WHO Grade II meningioma also showing fluorescing adjacent cortex, and histopathological examination revealed tumor cells in this brain tissue. Nevertheless, drawing a final conclusion as to whether PpIX fluorescence of the adjacent cortex is caused by leakage or tumor infiltration will require further systematic studies.

In our study, we further correlated PpIX fluorescence in the adjacent cortex with the meningioma arachnoid status. It is of note that we observed a significantly higher proportion of PpIX fluorescence within the adjacent cortex in cases with a disrupted arachnoid layer than in cases with an intact arachnoid layer. To the best of our knowledge, this is the first study that correlated the meningioma arachnoid status with PpIX fluorescence in the adjacent cortex.

\section{Surgical Benefits of 5-ALA-Induced Fluorescence in Intracranial Meningiomas}

According to our experience in more than 200 intracranial meningiomas, the use of 5-ALA represents a powerful technique for visualizing tumor tissue in the vast majority of such cases by means of visible PpIX fluorescence. With regard to maximizing the extent of resection, we found that PpIX fluorescence supports the neurosurgeon in identifying residual meningioma tissue at many, but not all, analyzed surgical sites. First of all, PpIX fluorescence is not helpful for visualizing the dural tail; we did not see fluorescence within the dural tail in any case, although histopathological analysis demonstrated the presence of tumor cells within dural tail specimens in some of the cases. Second, PpIX fluorescence is useful for identifying satellite lesions in the proximity of the meningioma that are barely recognizable with conventional white light alone. In the future, with the application of an angled endoscope that is capable of visualizing PpIX fluorescence, we expect to be able to detect additional satellite lesions further from the meningioma. ${ }^{8}$ Third, PpIX fluorescence is beneficial for visualizing the infiltrated bone flap adjacent to the meningioma; we recommend removing this fluorescing bone flap and replacing it by means of a cranioplasty. In contrast, PpIX fluorescence is not helpful in most sphenoorbital meningiomas, given that we did not observe visible PpIX fluorescence within the infiltrated bone in the majority of cases. Finally, according to our data PpIX fluorescence unfortunately cannot serve as an immediate intraoperative marker for high-grade meningiomas.

\section{Limitations}

This study has specific drawbacks. 1) We did not systematically analyze the presence of tumor cells within the dural tail or the adjacent cortex. However, none of the investigated dural tails showed PpIX fluorescence, and thus we did not systematically collect separate samples for histopathological analysis. Furthermore, due to ethical concerns we did not systematically collect tissue samples from the adjacent cortex based only on the presence of PpIX fluorescence. 2) We did not perform a systematic histopathological correlation of tissue samples derived from intratumoral areas with different PpIX fluorescence qualities (strong, vague, or no fluorescence). However, the main focuses of this exploratory study were to evaluate different PpIX fluorescence patterns and surgical benefits of PpIX fluorescence in meningiomas. A systematic histopathological correlation of different levels of PpIX fluorescence with the presence of tumor cells should be performed as a next step in future studies to calculate data on sensitivity and specificity. 3) We did not analyze all possible sites of remaining meningioma tissue, such as large sinuses (e.g., superior sagittal sinus). Visualization of residual meningioma tissue behind the lateral sinus wall, however, represents a current limitation of the 5-ALA technology. 4) Because the only bone flaps that we analyzed for PpIX fluorescence were those from cases in which preoperative imaging had shown evidence of tumor infiltration, we cannot exclude the possibility that PpIX fluorescence might also enable identification of meningioma infiltration in other cases (i.e., those in which preoperative imaging did not show evidence of bone invasion). 5) Additionally, 
the intraoperative semiquantitative classification of PpIX fluorescence as strong, vague, or none is subject to the neurosurgeon's perception. To overcome this limitation, quantitative assessment of PpIX accumulation in meningiomas to allow a more objective intraoperative fluorescence evaluation was recently proposed.,35 6) Finally, we did not perform a follow-up analysis of the meningioma cases to investigate the rate of tumor recurrence. However, the aim of this study was to investigate the surgical benefits of PpIX fluorescence in meningiomas and thus the analysis of the meningioma recurrence rate during postoperative follow-up was not within the scope of this study, and this topic should be addressed in future.

\section{Conclusions}

This study represents a first large-scale systematic analysis of the surgical benefits of 5-ALA in the resection of intracranial meningiomas. According to our data, most of these tumors display a strong fluorescence signal with a homogeneous fluorescence pattern. Consequently, this technique might, in the future, help to reduce the risk of recurrence by enabling more "radical" resections of intracranial meningiomas through visualization of residual tumor tissue. Interestingly, PpIX fluorescence can also be observed within the adjacent cortex in a subgroup of patients. The significance of this finding and its impact on meningioma recurrence has to be clarified in further studies.

\section{Acknowledgments}

We thank Harald Heinzl, Department of Medical Computer Sciences, for statistical advice; Brigitta Hammer-Schmiedel for technical assistance; and Ingrid Dobsak for support with the figures.

\section{References}

1. Bekelis K, Valdés PA, Erkmen K, Leblond F, Kim A, Wilson $\mathrm{BC}$, et al: Quantitative and qualitative 5-aminolevulinic acidinduced protoporphyrin IX fluorescence in skull base meningiomas. Neurosurg Focus 30(5):E8, 2011

2. Borovich B, Doron Y: Recurrence of intracranial meningiomas: the role played by regional multicentricity. J Neurosurg 64:58-63, 1986

3. Borovich B, Doron Y, Braun J, Guilburd JN, Zaaroor M, Goldsher D, et al: Recurrence of intracranial meningiomas: the role played by regional multicentricity. Part 2: Clinical and radiological aspects. J Neurosurg 65:168-171, 1986

4. Chae MP, Song SW, Park SH, Park CK: Experience with 5 -aminolevulinic acid in fluorescence-guided resection of a deep sylvian meningioma. J Korean Neurosurg Soc 52:558-560, 2012

5. Coluccia D, Fandino J, Fujioka M, Cordovi S, Muroi C, Landolt H: Intraoperative 5-aminolevulinic-acid-induced fluorescence in meningiomas. Acta Neurochir (Wien) 152:1711-1719, 2010

6. Commins DL, Atkinson RD, Burnett ME: Review of meningioma histopathology. Neurosurg Focus 23(4):E3, 2007

7. Cornelius JF, Slotty PJ, Kamp MA, Schneiderhan T, Steiger HJ, El-Khatib M: Impact of 5-aminolevulinic acid fluorescence-guided surgery on the extent of resection of meningiomas-with special regard to high-grade tumors. Photodiagnosis Photodyn Ther 11:481-490, 2014

8. Cornelius JF, Slotty PJ, Stoffels G, Galldiks N, Langen KJ,
Steiger HJ: 5-Aminolevulinic acid and ${ }^{18} \mathrm{~F}$-FET-PET as metabolic imaging tools for surgery of a recurrent skull base meningioma. J Neurol Surg B Skull Base 74:211-216, 2013

9. David N, Louis MD, Cavenee WK, Ohgaki H, Wiestler OD: WHO Classification of Tumours of the Central Nervous System. Lyon, France: World Health Organization, 2007

10. Della Puppa A, Rustemi O, Gioffrè G, Troncon I, Lombardi $\mathrm{G}$, Rolma G, et al: Predictive value of intraoperative 5-aminolevulinic acid-induced fluorescence for detecting bone invasion in meningioma surgery. J Neurosurg 120:840-845, 2014

11. Della Puppa A, Scienza R: 5-aminolevulinic acid-guided resection of bone-invasive meningiomas. Neurosurg Focus 35(4):E6, 2013 (Letter)

12. Eicker SO, Floeth FW, Kamp M, Steiger H-J, Hänggi D: The impact of fluorescence guidance on spinal intradural tumour surgery. Eur Spine J 22:1394-1401, 2013

13. Eljamel MS: Which intracranial lesions would be suitable for 5-aminolevulenic acid-induced fluorescence-guided identification, localization, or resection? A prospective study of 114 consecutive intracranial lesions. Clin Neurosurg 56:93-97, 2009

14. Goyal N, Kakkar A, Sarkar C, Agrawal D: Does bony hyperostosis in intracranial meningioma signify tumor invasion? A radio-pathologic study. Neurol India 60:50-54, 2012

15. Hefti M, Holenstein F, Albert I, Looser H, Luginbuehl V: Susceptibility to 5-aminolevulinic acid based photodynamic therapy in WHO I meningioma cells corresponds to ferrochelatase activity. Photochem Photobiol 87:235-241, 2011

16. Kajimoto Y, Kuroiwa T, Miyatake S, Ichioka T, Miyashita $\mathrm{M}$, Tanaka $\mathrm{H}$, et al: Use of 5-aminolevulinic acid in fluorescence-guided resection of meningioma with high risk of recurrence. Case report. J Neurosurg 106:1070-1074, 2007

17. Kawahara Y, Niiro M, Yokoyama S, Kuratsu J: Dural congestion accompanying meningioma invasion into vessels: the dural tail sign. Neuroradiology 43:462-465, 2001

18. Marbacher S, Klinger E, Schwyzer L, Fischer I, Nevzati E, Diepers M, et al: Use of fluorescence to guide resection or biopsy of primary brain tumors and brain metastases. Neurosurg Focus 36(2):E10, 2014

19. Masubuchi T, Kajimoto Y, Kawabata S, Nonoguchi N, Fujishiro T, Miyatake S, et al: Experimental study to understand nonspecific protoporphyrin IX fluorescence in brain tissues near tumors after 5-aminolevulinic acid administration. Photomed Laser Surg 31:428-433, 2013

20. Millesi M, Kiesel B, Woehrer A, Hainfellner JA, Novak K, Martínez-Moreno M, et al: Analysis of 5-aminolevulinic acid-induced fluorescence in 55 different spinal tumors. Neurosurg Focus 36(2):E11, 2014

21. Morofuji Y, Matsuo T, Hayashi Y, Suyama K, Nagata I: Usefulness of intraoperative photodynamic diagnosis using 5-aminolevulinic acid for meningiomas with cranial invasion: technical case report. Neurosurgery 62:102-104, 2008

22. Motekallemi A, Jeltema H-R, Metzemaekers JDM, van Dam GM, Crane LMA, Groen RJM: The current status of 5-ALA fluorescence-guided resection of intracranial meningiomasa critical review. Neurosurg Rev 38:619-628, 2015

23. Obwegeser A, Jakober R, Kostron H: Uptake and kinetics of ${ }^{14} \mathrm{C}$-labelled meta-tetrahydroxyphenylchlorin and 5-aminolaevulinic acid in the C6 rat glioma model. Br J Cancer 78:733-738, 1998

24. Oya S, Kawai K, Nakatomi H, Saito N: Significance of Simpson grading system in modern meningioma surgery: integration of the grade with MIB-1 labeling index as a key to predict the recurrence of WHO Grade I meningiomas. J Neurosurg 117:121-128, 2012

25. Pettersson-Segerlind J, Orrego A, Lönn S, Mathiesen T: Long-term 25-year follow-up of surgically treated parasagittal meningiomas. World Neurosurg 76:564-571, 2011 
26. Pieper DR, Al-Mefty O, Hanada Y, Buechner D: Hyperostosis associated with meningioma of the cranial base: secondary changes or tumor invasion. Neurosurgery 44:742-747, 1999

27. Rokni-Yazdi H, Azmoudeh Ardalan F, Asadzandi Z, Sotoudeh H, Shakiba M, Adibi A, et al: Pathologic significance of the "dural tail sign". Eur J Radiol 70:10-16, 2009

28. Simpson D: The recurrence of intracranial meningiomas after surgical treatment. J Neurol Neurosurg Psychiatry 20:22-39, 1957

29. Stummer W, Novotny A, Stepp H, Goetz C, Bise K, Reulen HJ: Fluorescence-guided resection of glioblastoma multiforme by using 5-aminolevulinic acid-induced porphyrins: a prospective study in 52 consecutive patients. J Neurosurg 93:1003-1013, 2000

30. Stummer W, Pichlmeier U, Meinel T, Wiestler OD, Zanella F, Reulen HJ: Fluorescence-guided surgery with 5-aminolevulinic acid for resection of malignant glioma: a randomised controlled multicentre phase III trial. Lancet Oncol 7:392401, 2006

31. Stummer W, Stepp H, Möller G, Ehrhardt A, Leonhard M, Reulen HJ: Technical principles for protoporphyrin-IX-fluorescence guided microsurgical resection of malignant glioma tissue. Acta Neurochir (Wien) 140:995-1000, 1998

32. Stummer W, Stocker S, Novotny A, Heimann A, Sauer O, Kempski $O$, et al: In vitro and in vivo porphyrin accumulation by C6 glioma cells after exposure to 5-aminolevulinic acid. J Photochem Photobiol B 45:160-169, 1998

33. Stummer W, Stocker S, Wagner S, Stepp H, Fritsch C, Goetz $\mathrm{C}$, et al: Intraoperative detection of malignant gliomas by 5-aminolevulinic acid-induced porphyrin fluorescence. Neurosurgery 42:518-526, 1998

34. Tokumaru A, O’uchi T, Eguchi T, Kawamoto S, Kokubo T, Suzuki M, et al: Prominent meningeal enhancement adjacent to meningioma on Gd-DTPA-enhanced MR images: histopathologic correlation. Radiology 175:431-433, 1990

35. Valdés PA, Bekelis K, Harris BT, Wilson BC, Leblond F, Kim A, et al: 5-Aminolevulinic acid-induced protoporphyrin IX fluorescence in meningioma: qualitative and quantitative measurements in vivo. Neurosurgery 10 (Suppl 1):74-83, 2014

36. van Alkemade H, de Leau M, Dieleman EMT, Kardaun JWPF, van Os R, Vandertop WP, et al: Impaired survival and long-term neurological problems in benign meningioma. Neuro Oncol 14:658-666, 2012

37. von Campe G, Moschopulos M, Hefti M: 5-Aminolevulinic acid-induced protoporphyrin IX fluorescence as immediate intraoperative indicator to improve the safety of malignant or high-grade brain tumor diagnosis in frameless stereotactic biopsies. Acta Neurochir (Wien) 154:585-588, 2012

38. Whitson WJ, Valdés PA, Harris BT, Paulsen KD, Roberts DW: Confocal microscopy for the histological fluorescence pattern of a recurrent atypical meningioma: case report. Neurosurgery 68:E1768-E1773, 2011
39. Widhalm G, Kiesel B, Woehrer A, Traub-Weidinger T, Preusser M, Marosi C, et al: 5-Aminolevulinic acid induced fluorescence is a powerful intraoperative marker for precise histopathological grading of gliomas with non-significant contrast-enhancement. PLoS One 8:e76988, 2013

40. Widhalm G, Minchev G, Woehrer A, Preusser M, Kiesel B, Furtner J, et al: Strong 5-aminolevulinic acid-induced fluorescence is a novel intraoperative marker for representative tissue samples in stereotactic brain tumor biopsies. Neurosurg Rev 35:381-391, 2012

41. Widhalm G, Wolfsberger S, Minchev G, Woehrer A, Krssak M, Czech T, et al: 5-Aminolevulinic acid is a promising marker for detection of anaplastic foci in diffusely infiltrating gliomas with nonsignificant contrast enhancement. Cancer 116: $1545-1552,2010$

42. Wilbers E, Hargus G, Wölfer J, Stummer W: Usefulness of 5-ALA (Gliolan ${ }^{\circledR}$ )-derived PPX fluorescence for demonstrating the extent of infiltration in atypical meningiomas. Acta Neurochir (Wien) 156:1853-1854, 2014

43. Wöhrer A, Waldhör T, Heinzl H, Hackl M, Feichtinger J, Gruber-Mösenbacher U, et al: The Austrian Brain Tumour Registry: a cooperative way to establish a population-based brain tumour registry. J Neurooncol 95:401-411, 2009

\section{Disclosures}

Stefan Wolfsberger reports serving as an educational consultant and a technological advisory board member for Medtronic. Mauricio Martínez-Moreno reports receipt of scholarship support from Conacyt (National Council of Sciences and Technology of Mexico).

\section{Supplemental Information \\ Previous Presentations}

Portions of this work were presented in poster form at the $83 \mathrm{rd}$ AANS Annual Scientific Meeting, Washington, DC, May 2-6, 2015.

\section{Author Contributions}

Conception and design: Knosp, Millesi, Wolfsberger, Widhalm. Acquisition of data: all authors. Analysis and interpretation of data: Knosp, Millesi, Wöhrer, Wolfsberger, Widhalm. Drafting the article: all authors. Critically revising the article: all authors. Reviewed submitted version of manuscript: all authors. Approved the final version of the manuscript on behalf of all authors: Knosp.

\section{Correspondence}

Engelbert Knosp, Department of Neurosurgery, Medical University of Vienna, Waehringer Guertel 18-20, Vienna 1097, Austria. email: engelbert.knosp@meduniwien.ac.at. 